\title{
The Guidance Algorithm of Air-to-Air Guided Missile with an Active Radar Target-Seeking Head to a Helicopter with Various Modes of its Flight
}

\author{
Alexander V. Bogdanov, Sergey A. Gorbunov, \\ Alexander A. Kuchin* and Ali A. Hadur \\ Military Academy of Aero-Space Defence \\ named after Marshal of Soviet Union G.K. Zhukov \\ Tver, Russian Federation
}

Received 10.09.2020, received in revised form 23.09.2020, accepted 01.10.2020

Abstract. On the basis of the mathematical apparatus of the statistical theory of optimal control, the method and algorithm of guidance of an air-to-air guided missile with an active radar target-seeking head when aiming at a «helicopter» class air target with various modes of its flight, including when hovering have been developed. The synthesis has been carried out as a result of flight experimental studies of radar signals reflected from a real helicopter. Also, recommendations for ensuring continuous tracking of a helicopter in an active radar target-seeking head have been presented.

Keywords: helicopter, aircraft guided missile, guidance method, guidance algorithm, aerial target tracking, statistical theory of optimal control.

Citation: Bogdanov A.V., Gorbunov S.A., Kuchin A.A., Hadur A.A. The guidance algorithm of air-to-air guided missile with an active radar target-seeking head to a helicopter with various modes of its flight, J. Sib. Fed. Univ. Eng. \& Technol., 2020, 13(7), 829-842. DOI: 10.17516/1999-494X-0269

(C) Siberian Federal University. All rights reserved

This work is licensed under a Creative Commons Attribution-Non Commercial 4.0 International License (CC BY-NC 4.0).

* Corresponding author E-mail address: kuchin.a.a@gmail.com 


\title{
Алгоритм наведения управляемой ракеты класса «воздух-воздух» с активной радиолокационной головкой самонаведения на вертолет при различном характере его полета
}

\author{
А.В. Богданов, С.А. Горбунов, \\ А.А. Кучин, А.А. Хадур \\ Военная академия воздушно-космической оборонь \\ им. Маршала Советского Союза Г.К. Жукова \\ Российская Федерачия, Тверь
}

Аннотация. На основе математического аппарата статистической теории оптимального управления синтезированы оптимальные по минимуму локального функционала качества метод и алгоритм наведения авиационной управляемой ракеты класса «воздух-воздух» с активной радиолокационной головкой самонаведения при наведении на воздушную цель класса «вертолет» при различном характере его полета, в том числе при зависании. Синтез выполнен в результате летно-экспериментальных исследований радиолокационных сигналов, отраженных от реального вертолета. Представлены рекомендации по обеспечению бессрывного сопровождения вертолета в активной радиолокационной головке самонаведения.

Ключевые слова: вертолет, авиационная управляемая ракета, метод наведения, алгоритм наведения, сопровождение воздушной цели, статистическая теория оптимального управления.

Цитирование: Богданов, А.В. Алгоритм наведения управляемой ракеты класса «воздух-воздух» с активной радиолокационной головкой самонаведения на вертолет при различном характере его полета / А.В. Богданов, С.А. Горбунов, А.А. Кучин, А.А. Хадур // Журн. Сиб. федер. ун-та. Техника и технологии, 2020. 13(7). С. 829-842. DOI: $10.17516 / 1999-494 X-0269$

\section{Введение}

Опыт применения армейской авиации показал, что вертолеты являются мощным средством борьбы с артиллерией, бронетанковой и автотранспортной техникой $[1,2]$.

Расширение функций и областей применения вертолетов различного назначения предопределяет востребованность и актуальность разработки средств их поражения при решении тактических и оперативных задач. Одним из возможных способов борьбы с вертолетами является применение самолетов истребительной авиации [3, 4].

Применение управляемых ракет (УР) класса «воздух-воздух» с активной радиолокационной головкой самонаведения (АРГС), построенной по импульсно-доплеровскому принципу обработки в ней отраженных от воздушных целей (ВЦ) радиолокационных (РЛ) сигналов [3-6], позволит выполнять атаку вертолета по принципу «пустил-забыл».

Однако анализ существующих методов и алгоритмов наведения авиационной УР, синтезированных на основе статистической теории оптимального управления (СТОУ) в пространстве состояний, показал недостаточную их эффективность при наведении на вертолет при различном характере его полета, который может быть стационарным, полет с ускорением или тор- 
можением, полет в режиме «висение» [7-9]. Кроме того, методы и алгоритмы синтезированы без учета результатов экспериментальных исследований информационных свойств сигналов, отраженных от вертолета.

В работе под методом наведения понимаем закон формирования требуемой фазовой траектории, наведение по которой позволит поразить цель, а под алгоритмом - правило формирования управляющих сигналов, именуемых также параметрами рассогласования, по которому определяется несоответствие реальных фазовых координат хуі объекта управления (ОУ) их требуемым значениям $\mathbf{x}_{\mathbf{~} \mathbf{i}}(\mathrm{i}=1, \mathrm{r})[10-12]$.

Цель статьи - на основе СТОУ в пространстве состояний синтезировать оптимальные по минимуму локального функционала качества метод и алгоритм наведения авиационной УР с АРГС на вертолет при различном характере его полета с учетом результатов летно-экспериментальных исследований (ЛЭИ) информационных свойств РЛ-сигналов, отраженных от реального вертолета.

\section{Экспериментальные исходные данные}

В результате ЛЭИ информационных свойств РЛ-сигналов, отраженных от вертолета [7, 8, 13], сущность которых заключается в регистрации с помощью специальной аппаратуры, установленной в состав бортовой радиолокационной станции (РЛС) истребителя МиГ-31 сигналов, отраженных от реального вертолета при различном характере его полета, и их обработка в лабораторных условиях на основе использования узкополосного спектрального анализа, позволили сделать следующие выводы.

Во-первых, РЛ-сигнал, отраженный от вертолета, спектральный доплеровский портрет которого представлен на рис. 1, имеет сложную структуру, обусловленную отражениями сигнала от: фюзеляжа вертолета; вращающихся лопастей несущего винта, имеющих различную радиальную скорость; вращающихся частей силовой установки вертолета [7, 8, 14].

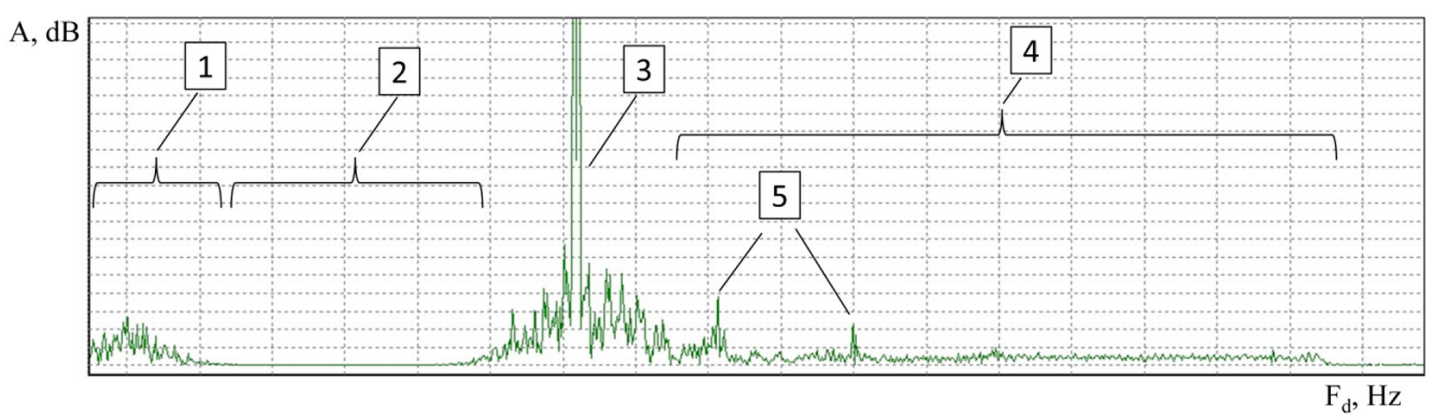

Рис. 1. Спектральный доплеровский портрет сигнала, отраженного от вертолета: 1 - отражения от подстилающей поверхности; 2 - зона режекции фильтра в бортовой РЛС истребителя (куда устанавливалась аппаратура регистрации); 3 - отражения от фюзеляжа вертолета; 4 - отражения от вращающихся лопастей несущего винта вертолета; 5 - отражения от вращающихся частей силовой установки вертолета

Fig. 1. Spectral Doppler portrait of the signal reflected from the helicopter: 1 - reflections from the underlying surface; 2 - rejection area; 3 - reflections from the helicopter fuselage; 4 -reflections from the rotor blades of the helicopter; 5 - reflections from the power plant of the helicopter 


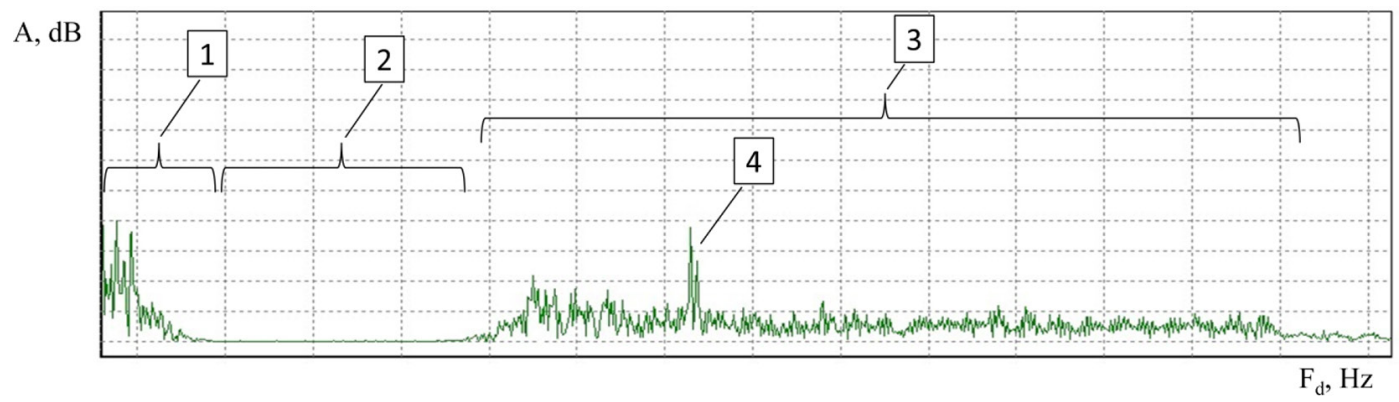

Рис. 2. Спектральный доплеровский портрет сигнала, отраженного от вертолета со скоростью менее 60 м/с: 1 - подстилающая поверхность; 2 - зона режекции; 3 - отражения от вращающихся лопастей несущего винта вертолета; 4 - отражения от вращающихся частей силовой установки вертолета

Fig. 2. Spectral Doppler portrait of the signal reflected from the helicopter at a speed of less than $60 \mathrm{~m} / \mathrm{s}: 1-$ reflections from the underlying surface; 2 - rejection area; 3 - reflections from the rotor blades of the helicopter; 4 - reflections from the power plant of the helicopter

Во-вторых, при полете вертолета со скоростью менее 216 км/ч $(60$ м/с) (в том числе и при полете в режиме «висение») отражения от фюзеляжа вертолета попадают в зону режекции, в этом случае будут наблюдаться (рис. 2) только отражения от вращающихся лопастей несущего винта вертолета и от вращающихся частей силовой установки вертолета.

Кроме того, известно [7, 8], что отражения РЛ-сигнала от вращающихся частей силовых установок летательных аппаратов имеют явную ракурсную зависимость. Поэтому для данной ситуации в качестве оценки скорости $V_{\text {сб }}$ сближения в режиме «висение» целесообразно использование значений доплеровских частот спектральных составляющих РЛ-сигнала, отраженного от вращающихся лопастей несущего винта вертолета $[7,8]$.

Таким образом, на основе анализа результатов ЛЭИ информационных свойств РЛсигналов, отраженных от вертолета, можно заключить, что как при стационарном полете (со значительной скоростью), так и при ускорении (торможении) вертолета целесообразным является использование при синтезе метода и алгоритма наведения УР с АРГС доплеровских частот, обусловленных скоростью сближения УР с фюзеляжем вертолета, а при полете в режиме «висение»- доплеровские частоты, обусловленные скоростями сближения УР с вращающимися лопастями несущего винта вертолета.

\section{Постановка задачи на синтез}

В общем случае задача синтеза метода и алгоритма наведения ракеты на воздушную цель в рамках СТОУ в пространстве состояний формулируется следующим образом [10, 11]: для системы с заданной частью

$$
\dot{\mathbf{x}}(t)=\mathbf{F}_{\mathbf{y}}(t) \mathbf{x}_{\mathbf{y}}(t)+\mathbf{B}_{\mathbf{y}}(t) \mathbf{u}(t)+\xi_{x}(t)
$$

предназначенной для отработки процесса

$$
\dot{\mathbf{x}}_{\mathbf{T}}(t)=\mathbf{F}_{\mathbf{T}}(t) \mathbf{x}_{\mathbf{T}}(t)+\xi_{\mathrm{T}}(t),
$$

при наличии измерений 


$$
\mathbf{z}(t)=\mathbf{H}(t) \mathbf{x}(t)+\xi_{\mathrm{H}}(t)
$$

найти закон управления

$$
\mathbf{u}(t)=\mathbf{K}^{-1} \mathbf{B}_{\mathbf{y}}^{\mathbf{T}} \mathbf{Q}\left[\hat{\mathbf{x}}_{\mathbf{T}}(t)-\hat{\mathbf{x}}_{\mathbf{y}}(t)\right],
$$

оптимальный по минимуму локального функционала качества

$$
I=\mathrm{M}_{\mathrm{y}}\left\{\left[\mathbf{x}_{\mathbf{r}}(t)-\mathbf{x}_{\mathbf{y}}(t)\right]^{\mathrm{T}} \mathbf{Q}\left[\mathbf{x}_{\mathbf{r}}(t)-\mathbf{x}_{\mathbf{y}}(t)\right]+\int_{0}^{t} \mathbf{u}^{\mathrm{T}}(t) \mathbf{K u}(t) d t\right\},
$$

где $\mathbf{F}_{\mathbf{y}}(t)$ - динамическая матрица состояния процесса $\dot{\mathbf{x}}_{\mathbf{y}}(t)$;

$\mathbf{x}_{\mathbf{y}}(t)$ - n-мерный вектор управляемых фазовых координат;

$\mathbf{B}_{\mathbf{y}}(t)$ - матрица эффективности управления;

$\mathbf{u}(t)$ - r-мерный вектор сигналов управления $(\mathrm{r} \leq \mathrm{n})$;

$\xi_{\mathrm{y}}(t)-\mathrm{n}$-мерный вектор центрированных гауссовских возмущений процесса $\dot{\mathbf{x}}_{\mathbf{y}}(t)$ с известной матрицей $\mathbf{G}_{\mathbf{y}}$ односторонних спектральных плотностей;

$\mathbf{F}_{\mathbf{T}}(t)$ - динамическая матрица состояния процесса $\dot{\mathbf{x}}_{\mathbf{T}}(t)$;

$\mathbf{x}_{\mathbf{T}}(t)$ - n-мерный вектор требуемых фазовых координат;

$\xi_{\mathrm{T}}(t)-\mathrm{n}$-мерный вектор центрированных гауссовских возмущений процесса $\dot{\mathbf{x}}_{\mathbf{T}}(t)$ с известной матрицей $\mathbf{G}_{\mathbf{\tau}}$ односторонних спектральных плотностей;

$\mathbf{H}(t)$ - матрица связи обобщенного вектора состояния $\mathbf{x}=\left[\begin{array}{ll}\mathbf{x}_{\mathbf{T}}^{\mathbf{T}} & \mathbf{x}_{\mathbf{y}}^{\mathbf{T}}\end{array}\right]^{\mathrm{T}}$ с m-мерным вектором наблюдений (измерений) $(\mathrm{m} \leq \mathrm{n})$;

$\xi_{\text {и }}(t)$ - n-мерный вектор центрированных гауссовских шумов измерений с известной матрицей $\mathbf{G}_{\mathbf{n}}$ односторонних спектральных плотностей;

$\mathbf{K}$ - матрица штрафов за величину сигналов управления;

$\mathbf{B}_{\mathbf{y}}^{\mathbf{T}}$ - матрица, определяющая способность системы воспринимать сигналы управления;

$\mathbf{Q}$ - матрица штрафов за точность управления $\left[\hat{\mathbf{x}}_{\mathbf{T}}(t)-\hat{\mathbf{x}}_{\mathbf{y}}(t)\right]$;

$\mathrm{M}_{\mathrm{y}}$ - математическое ожидание при условии, что имеются результаты измерения хотя бы части фазовых координат ху и $\mathbf{x}_{\mathbf{T}}$.

\section{Принятые допущения при синтезе метода и алгоритма}

1. Каналы управления ракетой в горизонтальной и вертикальной плоскости независимы, в дальнейшем рассматривается только горизонтальная плоскость, результаты ее рассмотрения могут быть применимы и для вертикальной плоскости.

2. При самонаведении на борту управляемой ракеты формируются значения требуемого поперечного ускорения.

3. УР и вертолет могут маневрировать с поперечными к линии визирования «УРвертолет» ускорениями $j_{\mathrm{p}}$ и $j_{\text {ц }}$ соответственно, а ускоряться - с продольным к линии визирования «УР-вертолет» ускорением $j_{\text {прод; }}$;

4. Под различным характером полета вертолета понимается любое из возможных его состояний:

- стационарный полет - горизонтальный полет вертолета в турбулентной атмосфере, при котором постоянные значения скорости $V_{\text {в }}$ высоты $H$ и курса не изменяются во времени; 
- ускорение (торможение) - полет вертолета, при котором постоянные значения скорости $V_{\text {в }}$ высоты Н и курса могут изменяться во времени;

- $\quad$ режим «висение» - полет вертолета, при котором скорость $V_{\text {в }} \approx 0 \mathrm{M} / \mathrm{c}$, а высота Н и курс постоянны во времени.

\section{Динамическая модель взаимного перемещения УР и вертолета при различном характере его полета}

На рис. 3 представлена схема взаимного расположения УР и вертолета (вид сверху).

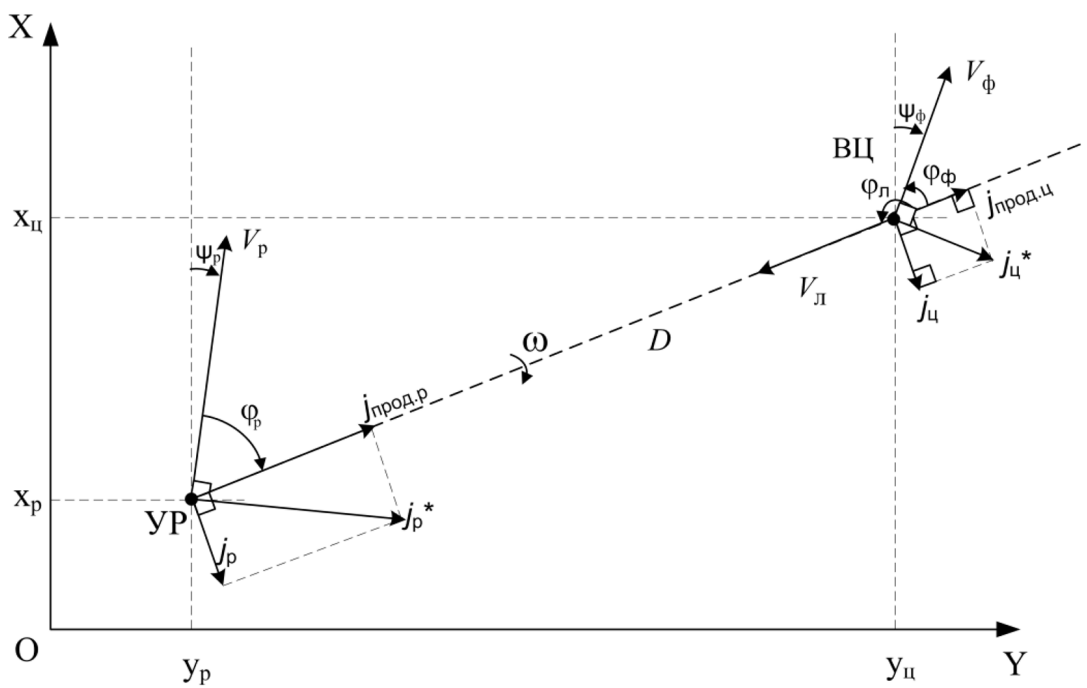

Рис. 3. Взаимное расположение УР и вертолета (вид сверху)

Fig. 3. The relative position of the rocket and the helicopter (top view)

На рис. 3 обозначено:

$D$ - расстояние между УР и фюзеляжем вертолета ВЦ класса «вертолет»;

$\omega-$ угловая скорость вращения линии визирования между УР и вертолетом;

$V_{\mathrm{p}}$ - текущая скорость УР;

$j_{\text {прод.p }}$ - продольное к линии визирования ускорение УР;

$j_{\mathrm{p}}-$ поперечное к линии визирования ускорение УР;

$\varphi_{\mathrm{p}}$ - текущее значение бортового пеленга вертолета с УР;

$\psi_{\mathrm{p}}$ - текущее значение курса полета УР;

$V_{\phi}$ - скорость перемещения фюзеляжа вертолета;

$V_{\text {л }}$-линейная скорость вращения наступающей лопасти несущего винта вертолета;

$j_{\text {прод.ц }}-$ продольное к линии визирования ускорение вертолета;

$j_{\text {ц }}$ поперечное к линии визирования ускорение вертолета;

$\varphi_{\phi}-$ угол между линией визирования и линией пути фюзеляжа вертолета;

$\varphi_{л}-$ угол между линией визирования и вектором линейной скорости вращения наступающей лопасти несущего винта вертолета $\left(\approx 180^{\circ}\right)$; 
$\left(\mathrm{x}_{\text {ц }}, \mathrm{y}_{\text {ц }}\right)$ - координаты вертолета (цели);

$\left(\mathrm{x}_{\mathrm{p}}, \mathrm{y}_{\mathrm{p}}\right)$ - координаты УР.

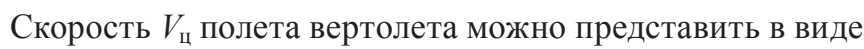

$$
V_{\mathrm{IL}}=V_{\mathrm{\pi}} k-V_{\phi} \cos \varphi_{\phi},
$$

где $k$ - коэффициент, компенсирующий «ошибочное» принятие скорости лопасти за скорость вертолета в целом, определяется по формуле

$$
k=\frac{1}{V_{\text {л }}}=\frac{2}{\left(F_{\text {дл }}-F_{\text {др }}\right) \lambda},
$$

где $F_{\text {др }}$ - частота Доплера, обусловленная собственной скоростью управляемой ракеты;

$F_{\text {дл }}-$ частота Доплера, обусловленная собственной линейной скоростью вращения наступающей лопасти несущего винта вертолета управляемой ракеты;

$\lambda$ - длина волны.

Проецируя скорости $V_{\mathrm{p}}$ и $V_{\text {ц }}$ на линию визирования и нормаль к ней, получаем

$$
\dot{D}=-V_{\text {ц }}-V_{\mathrm{p}} \cos \varphi_{\mathrm{p}} .
$$

Подставляя (6) в (8), получаем

$$
D \omega=V_{\text {л }}-V_{\phi} \sin \varphi_{\phi}+V_{\mathrm{p}} \sin \varphi_{\mathrm{p}} .
$$

Для упрощения примем допущение, что косинусы всех углов примерно равны единице, а синусы - своим аргументам.

Дифференцируя (9) по времени при $V_{\mathrm{p}}=$ const получаем

$$
\dot{D} \omega+D \dot{\omega}=\dot{V}_{\text {л }}-\dot{V}_{\phi} \varphi_{\phi}-V_{\phi} \dot{\varphi}_{\phi}+V_{\mathrm{p}} \dot{\varphi}_{\mathrm{p}}
$$

или

$$
\dot{\omega}=\frac{\dot{V}_{\text {л }}-\dot{V}_{\phi} \varphi_{\phi}}{D}-\frac{V_{\phi} \dot{\varphi}_{\phi}+V_{\mathrm{p}} \dot{\varphi}_{\mathrm{p}}}{D}-\frac{\dot{D} \omega}{D} .
$$

Так как $V_{\mathrm{p}} \dot{\varphi}_{\mathrm{p}}=j_{\mathrm{p}}, V_{\phi} \dot{\varphi}_{\phi}=j_{\phi}$, а $\dot{V}_{\pi}-\dot{V}_{\phi} \varphi_{\phi}=j_{\text {продш }}$, выражение (11) можно записать в виде

$$
\dot{\omega}=\frac{1}{D}\left(j_{\mathrm{p}}+j_{\text {прод ц }}-j_{\phi}\right)-\frac{\dot{D}}{D} \omega .
$$

В случае, когда $V_{\phi} \approx 0$ (режим «висение»),

$$
\dot{\omega}=\frac{1}{D}\left(j_{\mathrm{p}}+j_{\text {прод ц }}\right)-\frac{\dot{D}}{D} \omega,
$$

где $j_{\text {продш }}=\dot{V}_{\text {л }}$.

Для определения изменения бортового пеленга вертолета в процессе наведения на него вертолета представим формулу (9) как

$$
\begin{array}{r}
\varphi_{\mathrm{p}}=\arcsin \left(\frac{D \omega-V_{\mathrm{\Omega}}+V_{\phi} \sin \varphi_{\phi}}{V_{\mathrm{p}}}\right) . \\
-835-
\end{array}
$$


Дифференцируя (14) по времени при $V_{\mathrm{p}}=\mathrm{const}$, получаем

$$
\dot{\varphi}=\omega+\frac{1}{\dot{D}}\left(j_{\mathrm{p}}+j_{\text {прод п }}-j_{\phi}\right) .
$$

Таким образом, на основании формул (12) и (15) динамическая модель, описывающая взаимное перемещение УР и вертолета при различном характере его полета, имеет следующий вид:

$$
\begin{aligned}
& \begin{cases}\dot{\varphi}=\omega+\frac{1}{\dot{D}}\left(j_{\mathrm{p}}+j_{\text {прод ц }}-j_{\phi}\right)+\xi_{\varphi} ; & \varphi(0)=\varphi_{0} ; \\
\dot{\omega}=\frac{1}{D}\left(j_{\mathrm{p}}+j_{\text {грод ц }}-j_{\phi}\right)-\frac{\dot{D}}{D} \omega+\xi_{\omega} ; & \omega(0)=\omega_{0} ;\end{cases} \\
& \begin{cases}\dot{D}=-V_{\text {рц }} ; & D(0)=D_{0} ; \\
\dot{V}_{\text {рщ }}=j_{\text {грод }} ; & V_{\text {рц }}(0)=V_{\text {рц0 }} ; \\
\dot{j}_{\text {прод }}=-\alpha j_{\text {прод }}+\xi_{j_{\text {прод }}} ; & j_{\text {прод }}(0)=j_{\text {прод } 0},\end{cases}
\end{aligned}
$$

где $\xi_{\mathrm{w}}, \xi_{\mathrm{j}}$ - центрированные гауссовские шумы, характеризующие случайные возмущения системы «УР-вертолет»; $\alpha$ - коэффициент, характеризующий маневренные свойства УР.

Данная динамическая модель взаимного перемещения УР и вертолета будет в дальнейшем положена в основу синтеза оптимального метода и алгоритма наведения УР на вертолет.

\section{Синтез метода и алгоритма наведения ракеты на вертолет}

Для синтеза закона управления ракетой необходимо иметь модель состояния (1), (2) и функционал качества в виде (5).

Исходя из того, что параметр управления - поперечное ускорение УР, вектор управления будет иметь вид

$$
\mathbf{u}=j_{\mathrm{p}}+j_{\text {прод ц }}-j_{\Phi} .
$$

Требуемыми параметрами для управления ракетой, учитывающими управление ракурсом РЛ наблюдения в АРГС ракеты отраженных от вертолета РЛ-сигналов и угловой скоростью вращения линии визирования «УР-вертолет», являются $\varphi_{\text {т }}$ и $\omega_{\text {т }}$ соответственно, т.е. вектор требуемых фазовых координат $\mathbf{x}_{\mathrm{T}}$ будет иметь вид

$$
\mathbf{x}_{\mathbf{r}}=\left[\begin{array}{c}
\varphi_{\mathrm{T}} \\
\omega_{\mathrm{T}}
\end{array}\right] .
$$

Управляемые параметры для системы самонаведения УР - $\varphi$ и $\omega$, т.е. вектор управляемых фазовых координат ху будет следующим:

$$
\mathbf{x}_{\mathbf{y}}=\left[\begin{array}{c}
\varphi \\
\omega
\end{array}\right] .
$$

Так как параметром управления служит боковое ускорение ракеты $j_{\mathrm{p}}$, а управляемыми параметрами - $\varphi$ и $\omega$, то матрицу эффективности сигналов управления $\mathrm{B}_{\mathrm{y}}$ образуют коэффициенты при векторе управления u в динамической модели (16): 


$$
\mathbf{B}_{\mathbf{y}}=\left[\begin{array}{c}
\frac{1}{\dot{D}} \\
\frac{1}{D}
\end{array}\right]
$$

Матрицы штрафов за точность наведения $\mathbf{Q}$ и величину сигналов управления $\mathbf{K}$ в формуле (4) с учетом размерности матриц (19)-(21) имеют вид

$$
\mathbf{Q}=\left[\begin{array}{cc}
q_{\varphi} & 0 \\
0 & q_{\omega}
\end{array}\right] ; \quad \mathbf{K}=k_{j} .
$$

В результате функционал качества (5) принимает вид

$$
I=M_{y}\left\{\left[\begin{array}{c}
\varphi_{\mathrm{T}}-\varphi \\
-\omega
\end{array}\right]^{\mathrm{T}}\left[\begin{array}{cc}
q_{\varphi} & 0 \\
0 & q_{\omega}
\end{array}\right]\left[\begin{array}{c}
\varphi_{\mathrm{T}}-\varphi \\
-\omega
\end{array}\right]+\int_{0}^{t} k_{j}\left(j_{\mathrm{p}}+j_{\text {трод ц }}-j_{\phi}\right)^{2} d t\right\} .
$$

Подставив (18)-(22) в (4), получим следующее соотношение:

или

$$
j_{\mathrm{p}}+j_{\text {прод ц }}-j_{\phi}=\frac{1}{k_{j}}\left[\begin{array}{c}
\frac{1}{\dot{D}} \\
\frac{1}{D}
\end{array}\right]^{\mathrm{T}}\left[\begin{array}{cc}
q_{\varphi} & 0 \\
0 & q_{\omega}
\end{array}\right]\left[\begin{array}{c}
\varphi_{\mathrm{T}}-\varphi \\
-\omega
\end{array}\right]=\frac{q_{\varphi}}{k_{j} \dot{D}}\left(\varphi_{\mathrm{T}}-\varphi\right)-\frac{q_{\omega}}{k_{j} D} \omega,
$$

$$
j_{\mathrm{p}}=\frac{1}{k_{j}}\left[\begin{array}{c}
\frac{1}{\dot{D}} \\
\frac{1}{D}
\end{array}\right]^{\mathrm{T}}\left[\begin{array}{cc}
q_{\varphi} & 0 \\
0 & q_{\omega}
\end{array}\right]\left[\begin{array}{c}
\varphi_{\mathrm{T}}-\varphi \\
-\omega
\end{array}\right]=\frac{q_{\varphi}}{k_{j} \dot{D}}\left(\varphi_{\mathrm{\tau}}-\varphi\right)-\frac{q_{\omega}}{k_{j} D} \omega-j_{\text {прод }}+j_{\dot{\Phi ̆}_{\text {U }}} .
$$

Выполнение при синтезе условий линейно-квадратично-гауссовской задачи $[10,11]$ позволяет заменить истинные значения фазовых координат векторов $\mathbf{x}_{\mathbf{T}}$ и $\mathbf{x}_{\mathbf{y}}$ их оптимальными оценками, тогда алгоритм траекторного управления имеет вид

$$
\begin{aligned}
& \Delta_{j}=j_{\mathrm{pr}}-\hat{j}_{\mathrm{p}}, \\
& j_{\mathrm{pт}}=\frac{q_{\varphi}}{k_{j} \hat{\dot{D}}}\left(\varphi_{\mathrm{T}}-\hat{\varphi}\right)-\frac{q_{\omega}}{k_{j} \hat{D}} \hat{\omega}-\hat{j}_{\text {продц }}+\hat{j}_{\phi} .
\end{aligned}
$$

где $\Delta_{j}$ - параметр рассогласования, определяющий несоответствие текущего $j_{\mathrm{p}}$ и требуемого $j_{\mathrm{pт}}$ значений поперечного ускорения ракеты.

Учитывая, что $V_{\phi} \dot{\varphi}_{\phi}=j_{\phi}$, а $\dot{V}_{\text {л }}-\dot{V}_{\phi} \varphi_{\phi}=j_{\text {продц }}$ выражение (27) в случае использования при наведении УР с АРГС доплеровских частот, обусловленных скоростью сближения УР с фюзеляжем вертолета, можно представить в виде

$$
j_{\mathrm{pT}}=\frac{q_{\varphi}}{k_{j} \hat{\dot{D}}}\left(\varphi_{\mathrm{T}}-\hat{\varphi}\right)-\frac{q_{\omega}}{k_{j} \hat{D}} \hat{\omega}+\hat{\dot{V}}_{\phi} \hat{\varphi}_{\phi}+\hat{V}_{\phi} \hat{\dot{\varphi}}_{\phi}
$$

а при полете в режиме «висение», когда при наведении используются доплеровские частоты, обусловленные скоростями сближения УР с вращающимися лопастями несущего винта вертолета,

$$
j_{\mathrm{pт}}=\frac{q_{\varphi}}{k_{j} \hat{\dot{D}}}\left(\varphi_{\mathrm{\tau}}-\hat{\varphi}\right)-\frac{q_{\omega}}{k_{j} \hat{D}} \hat{\omega}-\hat{\dot{V}}_{\mathrm{л}} .
$$


Для формирования сигналов управления в соответствии с алгоритмом (26) необходимо иметь оценки следующих координат:

- дальность $D$ «ракета-вертолет» и скорость ее изменения;

- пеленг ј вертолета с управляемой ракетой;

- угловая скорость ш вращения линии визирования «УР-вертолет»;

- поперечное ускорение $j_{\phi}$ фюзеляжа вертолета, либо продольное ускорение $j_{\text {прод.ц }}$ вертолета.

Анализ полученных моделей состояния, а также сравнительный анализ полученных метода и алгоритма наведения с алгоритмами наведения, представленными в [3, 4, 10-12], позволяют сделать следующий вывод: применение нового алгоритма траекторного управления при наведении ракеты на вертолет при различном характере его полета позволит обеспечить непрерывное наведение управляемой ракеты класса «воздух-воздух» с АРГС, независимо от того, измерима ли скорость вертолета в целом (доплеровская частота сигнала, отраженного от фюзеляжа) или только от вращающихся лопастей несущего винта вертолета. С этой целью необходимо обеспечить бессрывное сопровождение вертолета в АРГС по доплеровской частоте.

\section{Техническое решение для обеспечения бессрывного сопровождения вертолета}

Для эффективного функционирования синтезированного алгоритма наведения ракеты класса «воздух-воздух» с АРГС, определяемого выражением (27), в [15] предложено следующее техническое решение бессрывного сопровождения вертолета в АРГС ракеты.

На рис. 4 приведена блок-схема, поясняющая данное техническое решение, на рис. $5(a-e)-$ эпюры, поясняющие его.

Бессрывное сопровождение вертолета в АРГС ракеты заключается в следующем [15] .

На вход блока 1 БПФ (рис. 4) на промежуточной частоте с выхода приемника АРГС поступает сигнал $S(t)$ (рис. 5a), отраженный от вертолета, который подвергается узкополосной доплеровской фильтрации на основе процедуры БПФ и преобразуется в амплитудно-частотный спектр $S(f)$ (рис. $5 b)$, составляющие которого обусловлены отражениями сигнала от фюзеляжа $\mathrm{A}_{\phi}$ сопровождаемого вертолета и вращающихся лопастей его несущего винта $A_{i}$.

С выхода блока 1 БПФ (рис. 4) на первые входы пороговых устройств 2 и 3 поступают соответственно значения амплитуд $\mathrm{A}_{\phi}$ и $A_{i}$ амплитудно-частотного спектра $S(f)$ отраженного от

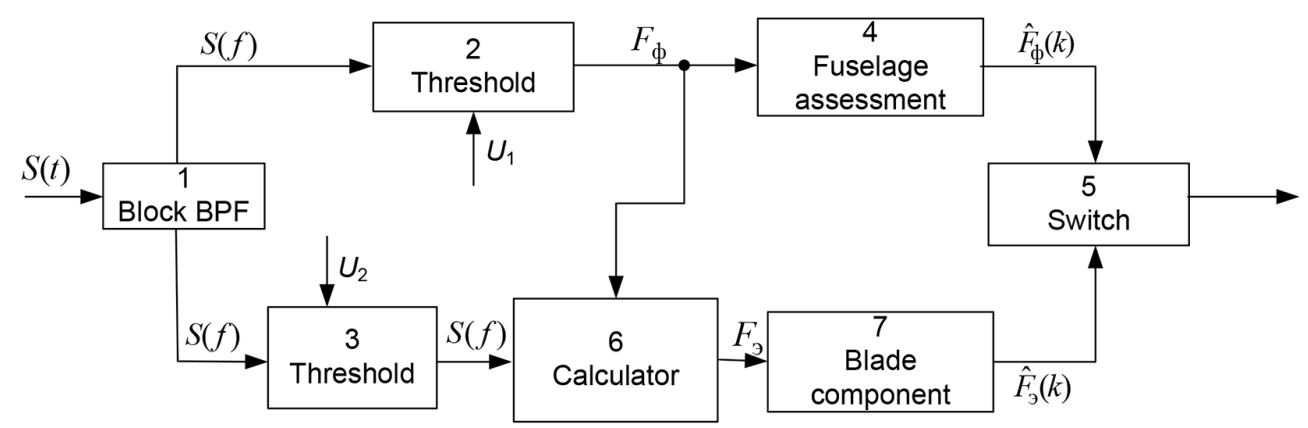

Рис. 4. Блок-схема

Fig. 4. Flowchart 
a)

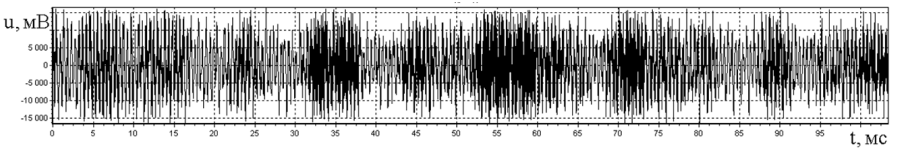

b)

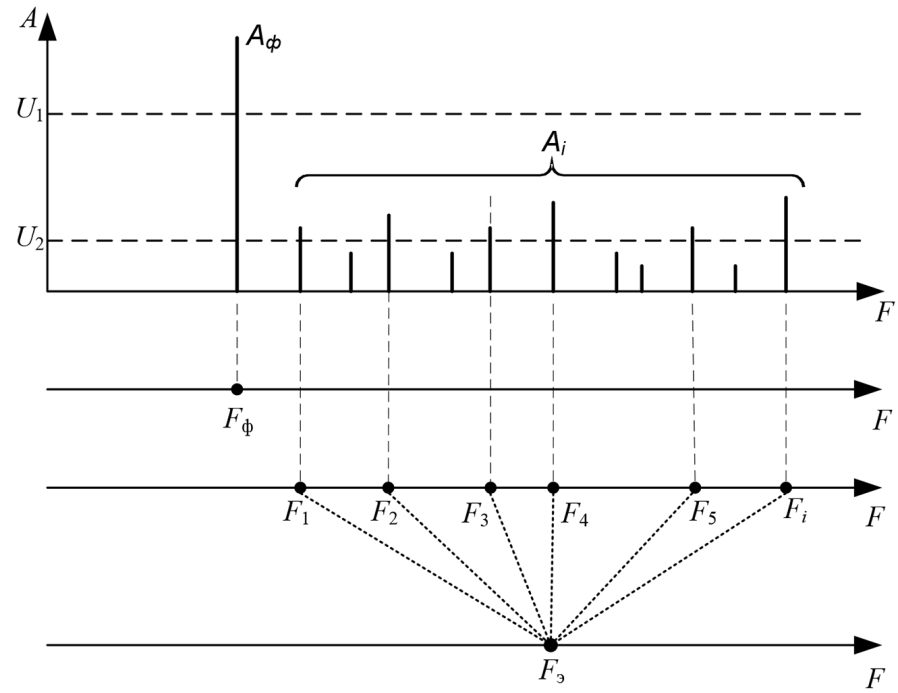

Рис. 5. Эпюры

Fig. 5. Diagrams

вертолета сигнала. На их вторые входы подаются значения порогов $U_{1}$ и $U_{2}$. Причем значение порога $U_{2}$ в $n$ раз меньше значения порога $U_{1}$.

В каждый $k$-й дискретный момент времени на выходе первого порогового устройства 2 определяется отсчет $F_{\phi}$ доплеровской частоты (рис. $5 c$ ) спектральной составляющей с максимальной амплитудой $\mathrm{A}_{\phi}$, превысившей первый порог $U_{1}$ и соответствующей отражениям сигнала от фюзеляжа вертолета, который подается (рис. 4) на вход первого фильтра 4-го сопровождения. В результате на его выходе формируется оценка $\hat{F}_{\phi}(k)$ доплеровской частоты, которая поступает на первый вход коммутатора 5.

Одновременно с определением отсчетов $F_{\phi}$ доплеровских частот, соответствующих отражениям сигнала от фюзеляжа вертолета, в каждый $k$-й дискретный момент времени определяются отсчеты $F_{i}$ амплитуд $\mathrm{A}_{i}$, превысивших второй порог $U_{2}$ и соответствующих отражениям сигнала от лопастей несущего винта вертолета (рис. $5 d$ ), за исключением спектральной составляющей с максимальной амплитудой $\mathrm{A}_{\phi}$ и соответствующей отражениям сигнала от фюзеляжа вертолета.

В вычислителе 6 (рис. 4) в соответствии с выражением

$$
F_{\ni}=\frac{M\left\{A_{i} F_{i}\right\}}{M\left\{A_{i}\right\}},
$$

где $\mathrm{M}\{\ldots\}$ - операция математического ожидания; $A_{i}-i$-я амплитуда спектральной составляющей сигнала, соответствующая отражениям сигнала от лопастей несущего винта вертолета,

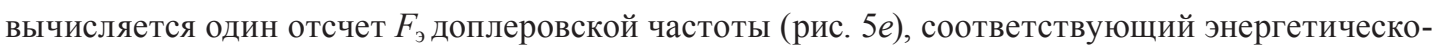


му центру отражений сигнала от лопастей несущего винта вертолета, который подается на вход второго фильтра 7 (рис. 4) сопровождения, где осуществляется фильтрация полученных

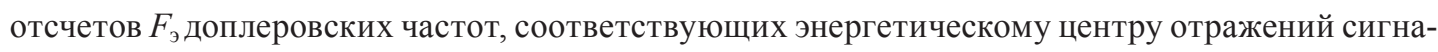
ла от лопастей несущего винта вертолета, в результате чего на его выходе формируется оценка $\hat{F}_{9}(k)$ доплеровской частоты по отражениям сигнала от лопастей его несущего винта, которая поступает на второй вход коммутатора 5.

На выходе коммутатора 5 при наличии отсчетов $F_{\phi}$ доплеровских частот спектральных составляющих, превысивших первый порог $U_{1}$ и соответствующих отражениям сигнала от фюзеляжа вертолета, формируется оценка $\hat{F}_{\phi}(k)$ доплеровской частоты с выхода первого фильтра 4 сопровождения вертолета по отражениям сигнала от его фюзеляжа, а при наличии отсчетов $F_{i}$ доплеровских частот спектральных составляющих, превысивших второй порог $U_{2}$ и не превысивших первый порог $U_{1}$ - оценка $\hat{F}_{9}(k)$ доплеровской частоты с выхода второго фильтра 7 сопровождения вертолета по отражениям сигнала от лопастей его несущего винта.

В результате обработки зарегистрированных реальных РЛ-сигналов, отраженных от различных типов отечественных вертолетов, установлено $[7,8,13]$ следующее:

амплитуда спектральной составляющей сигнала, отраженного от фюзеляжа вертолета $\mathrm{A}_{\phi}$, в 10-12 раз превышает амплитуды $\mathrm{A}_{i}$ спектральных составляющих сигнала, отраженного от лопастей несущего винта вертолета, то есть величина порога $U_{2}$ должна быть меньше в $10-12$ раз величины порога $U_{1}$;

диапазон доплеровских частот, занимаемый отражениями сигнала от лопастей несущего винта вертолета, составляет 8-12 кГц;

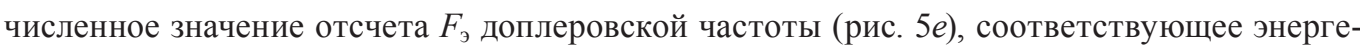
тическому центру отражений сигнала от лопастей несущего винта вертолета, вычисляемого в соответствии с выражением (30), отличается на $12-15 \%$ от значения отсчета доплеровской частоты, вычисленного в виде математического ожидания отсчетов доплеровских частот амплитуд $\mathrm{A}_{i}$, то есть точность оценки доплеровской частоты $\hat{F}_{9}(\mathrm{k})$ выше относительно точности оценки доплеровской частоты, получаемой на основе фильтрации отсчетов доплеровской частоты, соответствующих математическому ожиданию отсчетов доплеровских частот амплитуд $\mathrm{A}_{i}$.

\section{Выводы}

1. На основе СТОУ в пространстве состояний синтезированы оптимальные по минимуму локального функционала качества метод и алгоритм наведения авиационной УР с АРГС на вертолет при различном характере его полета.

2. Предложено техническое решение по бессрывному сопровождению вертолета при различном характере его полета с учетом результатов летно-экспериментальных исследований информационных свойств РЛ-сигналов, отраженных от него.

\section{Список литературы / References}

[1] Будник А.С. Опыт применения вертолетов армейской авиации в локальных войнах и вооруженных конфликтах. Военная мысль, 2016, 4, $22-26$ [Budnik A.S. Experience in the useofarmy aviation helicopters in local war sand wars armedconflicts. Military thought, 2016, 94, 22-26 (in Russian)] 
[2] Федосов Е.А. Авиация ПВО России и научно-технический прогресс: боевые комплексы и системы вчера, сегодня, завтра: монография. М.: Дрофа, 2004, 256 с. [Fedosov E.A. Russian air defense aviation and scientific and technical progress: combat complexes and systems of defense, today, tomorrow: monograph, Moscow: Bustard, 2004, 256 p. (in Russian)]

[3] Анциферов А.А., Богданов А.В., Бондарев В.Н., Гарин Е.Н., Гончаров А.М., Кучин А.А., Лютиков И.В. Синтез оптимальных алгоритмов наведения летательных аппаратов на групповую воздушную цель, Журнал Сибирского федерального университета. Техника и технологии, 2017, 10(2), 169-182 [Antsiferov A.A., Bogdanov A.V., Bondarev V.N., Garin E.N., Goncharov A.M., Kuchin A.A., Lytikov I.V. Synthesis of optimal algorithms for guiding aircraft to a group air target. Journal of Siberian Federal University. Engineering and technology, 2017, 10(2), 169-182 (in Russian)]

[4] Богданов А.В., Филонов А.А., Ковалев А.А., Кучин А.А. и др. Методы самонаведения истребителей и ракет класса «воздух-воздух» на групповую воздушную цель: монография, Красноярск: Сиб. федер. ун-т, 2014, 168 с. [Bogdanov A.V., Filonov A.A., Kovalev A.A., Kuchin A.A. Homing methods of fighters and air-to-air missales to a group air target: monograph, Krasnoyarsk: Siberian Federal University, 2014, 168 p. (in Russian)]

[5] Дудник П.И., Кондратенков Г.С., Татарский Б.Г., Ильчук А.Р., Герасимов А.А. Авиационные радиолокационные комплексы и системы, М.: ВВИА им. Н.Е. Жуковского, 2006, 1112 c. [Dudnik P.I., Kondratenkov G.S., Tatarsky B.G., Ilychuk A.R., Gerasimov A.A. Aviation radar complexes and systems, M.: VVIA im. N.E. Gukovsky, 2006, 1112 p. (in Russian)]

[6] Сколник М.И. Справочник по радиолокации. В 2 кн. Кн. 1., М.: Техносфера, 2014, 672 с. [Skolnik M.I. Radar Handbook. Vol. 1. In 2 volumes, M.: Tahnosfere, 2014, 672 p. (in Russian)]

[7] Богданов А.В., Васильев О.В., Каневский М.И., Коротков С.С., Миронович С.Я., Кучин А.А., Анциферов А.А., Закомолдин Д.В. Результаты летно-экспериментальных исследований по оценке возможностей всестороннего распознавания воздушных целей в импульсно-доплеровской РЛС воздушного базирования в сантиметровом диапазоне волн. Успехи современной радиоэлектроники, 2016, 11, 174-179 [Bogdanov A.V., Vasiliev O.V., Kanevskiy M.I., Korotkov S.S., Mironovich S.Y., Kuchin A.A., Antsiferov A.A., Zakomoldin D.V. The results of experimental studies on assessment of comprehensive air target recognition in centimeter band by airborne pulse Doppler radar, Advances in modern electronics, 2016, 11, 174-179 (in Russian)]

[8] Аганин А.Г. Метод синтеза многоэтапного когерентного алгоритма обнаружения на основе экстремальной статистики. Информационно-измерительные и управляющие системы, 2008, 6, 9, 40-49. [Aganin A.G. Method for synthesizing a multi-stage coherent detection algorithm based on extreme statistics. Information-measuring and control systems, 2008, 6, 9, 40-49 (in Russian)]

[9] Богданов А.В., Горбунов С.А., Кучин А.А., Шпортко С.А. Точностные и вероятностные характеристики алгоритма распознавания режима полета вертолета в радиолокационной системе воздушного базирования. Журнал Сибирского федерального университета. Техника и технологии, 2018, 11(3), 358-370 [Bogdanov A.V., Gorbunov S.A., Kuchin A.A., Shportko S.A. Accuracy and probabilistic characteristics of the helicopter flight mode recognition algorithm in an airborne radar system, Journal of Siberian Federal University. Engineering and technology, 2018, 11(3), 358-370 (in Russian)]

[10] Верба В.С. и др. Информационно-измерительные и управляющие радиоэлектронные системы и комплексы: монография. М.: Радиотехника, 2020, 490 с. [Verba V.S. Information-

$$
-841-
$$


measuring and control radio-electronic systems and complexes: monograph. Moscow: Radio engineering, 2020, 490 p. (in Russian)]

[11] Меркулов В.И., Верба В.С., Ильчук А.Р. Автоматическое сопровождение целей в РЛС интегрированных авиационных комплексов. Теоретические основы. РЛС в составе интегрированного авиационного комплекса. монография в 3 т. Т. 1. М.: Радиотехника, 2018, 316 с. [Merculov V.I., Verba V.S., Il'chuk A.R. Automatic-support of the central control system the radar of integrated aviation complexes. Theoretical bases. Radar as part of an integrated aviation complex, monograph in 3 vol. Vol. 1. Moscow: Radio engineering, 2018, 316 p. (in Russian)]

[12] Меркулов В.И., Верба В.С., Ильчук А.Р., Колтышев Е.Е. Автоматическое сопровождение целей в РЛС интегрированных авиационных комплексов. Сопровождение одиночных целей, монография в 3 т. T. 2. М.: Радиотехника, 2018, 486 c. [Merculov V.I., Verba V.S., Il'chuk A.R., Koltyshev E.E. Automatic-support of the central control system the radar of integrated aviation complexes. Support of single goals. Monograph in 3 vol. Vol. 2. Moscow: Radio engineering, 2018, 486 p. (in Russian)]

[13] Богданов А.В., Коротков С.С., Кучин А.А., Бондарев В.Н., Лютиков И.В. Концепция распознавания воздушных целей в авиационном радиолокационном комплексе. Журнал Сибирского федерального университета. Техника и технологии, 2016, 9(3), 319-331 [Bogdanov A.V., Korotkov S.S., Kuchin A.A., Bondarev V.N., Lytikov I.V. The concept of recognition of air targets in the aviation radar complex, Journal of Siberian Federal University. Engineering and technology, 2016, 9(3), 319-331 (in Russian)]

[14] Гейстер C.Р., Нгуен Т.Т. Математические модели радиолокационного сигнала, отраженного от несущего винта вертолета, в приложении к обращенному синтезу апертуры. Изв. вузов России. Радиоэлектроника, 2019, 22(3), 74-87 [Heister S.R., Thai T. Nguyen. Mathematical models of the radar signal reflected from a helicopter main rotor in application to inverse synthesis of antenna aperture. Journal of the Russian Universities. Radioelectronics. 2019, 22(3), 74-87 (in Russian)]

[15] Способ сопровождения вертолета в импульсно-доплеровской радиолокационной станции: патент 2728278, Российская Федерация. 2019, № 22. 2 с. [Helicopter tracking method in pulse-Doppler radar station: patent 2728278, Russian Federation. 2019, no. 22. 2 p. (in Russian)] 